\title{
In Vivo Evaluation of Leishmanicidal Activity of Benzophenone Derivatives by qPCR
}

Fabio Antonio Colombo ${ }^{1 *}$, Rayssa Azara Reis ${ }^{1}$, Juliana Barbosa Nunes¹, Danielle Ferreira Dias², Marcelo Henrique dos Santos², Claudio Viegas Junior ${ }^{3}$ and Marcos José Marques ${ }^{1}$

${ }^{1}$ Department of Pathology and Parasitology, Laboratory of Molecular Biology of Microorganisms, UNIFAL-MG, Rua Gabriel Monteiro da Silva, 700, 37130-000, Alfenas-MG, Brazil

${ }^{2}$ Laboratory of Phytochemistry and Medicinal Chemistry, UNIFAL-MG, Rua Gabriel Monteiro da Silva, 700, 37130-000, Alfenas-MG, Brazil

${ }^{3}$ Laboratory of Research in Medicinal Chemistry, Institute of Chemistry, UNIFAL-MG, Avenida Jovino Fernandes Sales, 2600, 37130-000, Alfenas-MG, Brazil

\begin{abstract}
In many tropical areas, infections by Leishmania species are endemic and include visceral leishmaniasis (VL), which is often fatal if untreated. Outside India, VL treatment and control are based on long-term administration of highly toxic pentavalent antimonials. Previously, we described the synthesis and in vitro leishmanicidal activity of a series of nine benzophenone derivatives with low toxicity towards murine macrophages. Here in, we report the in vivo evaluation of the most promising active compounds of that series in an experimental model of established VL by L. (L). infantum chagasi in hamsters. Importantly, parasite DNA (amastigote form) quantification in infected tissues was performed by real time PCR, for improved detection accuracy and speed. Compounds 2-Hydroxy-4O-(3,3-dimethyl)-allylbenzophenone (LFQM-117 (1)), 4-O-(3,3-Dimethyl)-allylbenzophenone (LFQM-120 (2)) and 4,4'-Di-methoxybenzophenone (LFQM-121 (3)) were administered as oral suspensions ( $50 \mathrm{mg} / \mathrm{kg} /$ day) for 10 days, after 50 days of parasite inoculation, and their efficacy was compared to pentavalent antimonial Glucantime (GLU). Compound 1 significantly reduced the number of parasites in the spleen $\left(1.64 \times 10^{2}\right.$ amastigotes $/ \mathrm{g}, \mathrm{vs} .1 .16 \times 10^{6}$ amastigotes/g in the untreated control), while compound 2 significantly reduced $(\mathrm{p}<0.05)$ the number of parasites in the liver $\left(1.28 \times 10^{4}\right.$ amastigotes $/ g$, vs. $1.76 \times 10^{5}$ amastigotes $/ g$ in the untreated control $)$ of infected animals. Glucantime was the most effective in the treatment of infected animals $\left(1.15 \times 10^{1}\right.$ and $3.20 \times 10^{2}$ amastigotes/g in the spleen and liver, respectively), but with higher toxicity then the most active compounds LFQM-117 (1) and LFQM-120 (2).
\end{abstract}

Keywords: Visceral leishmaniasis; In vivo activity; Benzophenones derivatives; qPCR; Treatment; Oral route

\section{Introduction}

Leishmaniasis are zoonosis caused by flagellated protozoan parasites from the genus Leishmania and are transmitted to humans by the bite of infected female phlebotomine sandflies. This group of diseases occurs mainly in tropical and subtropical regions of the planet, being endemic mostly in developing countries. Visceral Leishmaniasis (VL) is responsible for approximately 50,000 human deaths annually, with $90 \%$ of new cases of visceral leishmaniasis occurring in Bangladesh, Brazil, Ethiopia, India, South Sudan and Sudan [1,2]. VL produces a prolonged and irregular fever, anemia, and hepatosplenomegaly. The disease affects mainly the liver, spleen and bone marrow, but can also affects the lymph nodes, kidney, intestines, lungs and skin. If untreated, VL can be fatal. In the New World, VL (termed 'American VL') is caused by the species $L$. (L.) infantum chagasi, which infects both adults and children, and is transmitted by sand flies from the genus Lutzomyia [3]. Outside India, where resistance to pentavalent antimony compounds has emerged, treatment and control of VL are based on Glucantime and Pentostam use, which have limitations like long-term administration, high toxicity and unsafety for use in pregnant patients [4]. Benzophenone derivatives have shown promising in vitro leishmanicidal activity and a number of prototype candidates are under evaluation as lead compounds for the development of new drugs against leishmaniasis. In a recent study, we reported a series of nine alkyl-substituted benzophenone derivatives with increased leishmanicidal activity against promastigote form of L. amazonensis and reduced toxicity towards murine macrophages [5]. Our results suggested that the increased lipophilicity of these compounds could facilitate membrane permeation for drug entry into the parasite cells.

More recently, these benzophenone derivatives were further evaluated in vitro against amastigote form of L. amazonensis and compounds LFQM-117 (1), LFQM-120 (2) and LFQM-121 (3) showed the best relationship between their potency (IC50) and safety (SI), considering their effects towards amastigote forms and murine macrophages, respectively [6]. Despite their promising activity and low toxicity in vitro, these compounds have not been tested in vivo to evaluate their effectiveness in controlling or treating the disease in animal models of experimental leishmaniasis. Then, here in, we report the evaluation of the most active and promising hydroxy-alkylbenzophenone derivatives 1-3 described previously [5,6], in an in vivo model of leishmaniasis by $L$. (L.) infantum chagasi in hamsters. To improve treatment monitoring, the detection and quantification of amastigote forms was performed by quantitative real-time PCR (qPCR). When compared to conventional PCR, qPCR represents a more efficient, sensitive and robust technique for the detection of amastigotes RNA in various tissues (spleen, skin, lymph, blood and bone marrow) and in different clinical groups [7-9]. In addition, qPCR is currently considered the most reliable method to evaluate the in vivo activity of compounds against visceral leishmaniasis, allowing effective disease monitoring, with increased speed and accuracy compared with standard methods $[10,11]$.

*Corresponding author: Fabio Antonio Colombo, Faculty of Pharmaceutical Sciences, Department of Pathology and Parasitology, University Federal of Alfenas, PO Box 37130-000, Alfenas, Brazil, Tel: +5503537019534; E-mail: colombofabio@uol.com.br

Received May 04, 2017; Accepted May 18, 2017; Published May 25, 2017

Citation: Colombo FA, Reis RA, Nunes JB, Dias DF, dos Santos MH, et al. (2017) In Vivo Evaluation of Leishmanicidal Activity of Benzophenone Derivatives by qPCR. Med Chem (Los Angeles) 7: 890-893. doi: 10.4172/2161-0444.1000448

Copyright: (c) 2017 Colombo FA, et al. This is an open-access article distributed under the terms of the Creative Commons Attribution License, which permits unrestricted use, distribution, and reproduction in any medium, provided the original author and source are credited. 


\section{Materials and Methods}

\section{Compounds}

In this study, it was evaluated three benzophenone derivatives 2-Hydroxy-4-O-(3,3-dimethyl)-allylbenzophenone (LFQM-117, 1), 4-O-(3,3-Dimethyl)-allylbenzophenone (LFQM-120, 2) and 4,4'-Dimethoxybenzophenone (LFQM-121, 3, Table 1). These compounds were prepared from the commercial 2,4-dihydroxybenzophenone, 4-hydroxybenzophenone and 4,4'-dihydroxybenzophenone, respectively, as previously reported [5,6]. Briefly, the target compounds were performed using the method described by Yang et al. with the chemical precursors being subjected to alkylation in DMF/acetone, in the presence of $\mathrm{K}_{2} \mathrm{CO}_{3}$ and an alkylating reagent. Compound characterization was performed by IR and NMR [5]. We choose Glucantime as reference drug because it is the only compound allowed to human treatment in Brazil, but the Miltefosine it is not available to humans only to dogs $[12,13]$.

\section{Parasites and animals}

Leishmania (L.) infantum chagasi (strain MHOM/BR/1972/BH46) promastigotes were maintained in M-199 medium supplemented with $10 \%$ calf serum and $0.25 \%$ hemin, at $24^{\circ} \mathrm{C}$. Amastigotes were maintained by passaging in female golden hamsters (Mesocricetus auratus). Animals were kept in sterile absorbent material boxes, with food and water ad libitum, and in natural light/dark cycle. All experimental procedures involving animals were approved by the Research Ethics Commission of the Federal University of Alfenas (project number 394/2012), and were performed according to the Guide for the Care and Use of Laboratory Animals [14]. For experimental infections, the spleen of infected animals was removed and macerated using a tissue grinder and the number of amastigotes was determined as described previously.

\section{In vivo testing of experimental compounds against Leishmania}

Female golden hamsters that had been recently weaned $(120$ g) were infected intraperitoneally with $1 \times 10^{7}$ amastigotes of $L$. (L.) infantum chagasi (MHOM/BR/1972/BH46) and maintained in sterile absorbent material boxes, with water and food ad libitum. In the chronic phase (around 50 days of infection), animals were divided into 5 groups ( $n=4$ /group), and subjected, for 10 consecutive days, to one of the following treatments: $0.5 \%$ of carboxymethyl cellulose (vehicle) suspension, administered orally (untreated, or UTG group); $50 \mathrm{mg} / \mathrm{kg} /$ day of Glucantime (GLU), by intraperitoneal injection (GLU group); 50 $\mathrm{mg} / \mathrm{kg} /$ day of compounds 1,2 or 3 , administered orally as suspensions in $0.5 \%$ of carboxymethyl cellulose. After 10 days of treatment, animals were sacrificed in a $\mathrm{CO}_{2}$ chamber, and a sample of the spleen and the liver (approximately $50 \mathrm{mg}$ ) was removed, weighed and used for total RNA extraction, as previously described [9].

\section{DNA extraction}

Standard curves of parasite DNA for use in quantitative real-time PCR (qPCR) experiments were produced as described previously [8]. Briefly, promastigotes from stationary phase cultures were harvested by centrifugation at $1000 \mathrm{~g}$ for 10 minutes, washed twice in PBS ( $\mathrm{pH}$ 7.2), and counted in a hemocytometer. Then, parasites were re-suspended in PBS ( $\mathrm{pH} 7.2$ ) for a concentration of $1 \times 10^{8}$ cells $/ \mathrm{mL}$, and serially diluted (1:10) up to a concentration of $1 \times 10^{2}$ cells $/ \mathrm{mL}$ (in triplicates). Lysis buffer (10 mM Tris- $\mathrm{HCl}, \mathrm{pH}$ 8.0, containing $10 \mathrm{mM}$ EDTA, 0.5\% SDS, $0.01 \% \mathrm{~N}$-Lauroylsarcosine sodium salt, $100 \mathrm{ug} / \mathrm{mL}$ Proteinase K) was then added to parasite suspensions, at a ratio of $1: 4(\mathrm{v} / \mathrm{v})$, samples were mixed by vortexing and incubated at $56^{\circ} \mathrm{C}$, for 40 minutes. DNA was extracted from lysed samples using the QIAamp DNA extraction Mini Kit (Qiagen), according to the manufacturer's instructions.

\section{RNA extraction and cDNA synthesis}

Fragments of liver and spleen ( $\sim 50 \mathrm{mg}$; weighed using sterile and disposable surgical material) removed from treated mice were placed in sterile microfuge tubes and frozen immediately at $-80^{\circ} \mathrm{C}$ in storage buffer (RNAlater). RNA extraction was performed 24 hours after fragment removal, using the RNeasy Mini Kit (Qiagen), according to the manufacturer's instructions. RNA samples were frozen immediately after extraction. For reverse-transcription into cDNA, $1 \mu \mathrm{l}$ of dNTPs mix $(10 \mathrm{mM})$ and $1 \mu \mathrm{l}$ of random primers $(3 \mu \mathrm{g} / \mu \mathrm{L})$ were added to 11 $\mu \mathrm{l}$ of RNA sample, and samples were incubated in a thermal cycler for approximately 5 minutes, at $65^{\circ} \mathrm{C}$. Then, tubes were placed on ice for 20 $\mathrm{s}$, and $2 \mu \mathrm{l}$ of DTT $(100 \mathrm{mM}$ ) and $4 \mu \mathrm{l} 5 \mathrm{x}$ buffer (Tris-HCl $250 \mathrm{mM}, \mathrm{pH}$ 8.3 , containing $375 \mathrm{mM} \mathrm{KCl}, 15 \mathrm{mM} \mathrm{MgCl}_{2}$ ) were added, and samples were incubated again in the thermal cycler for $20 \mathrm{~s}$, at $37^{\circ} \mathrm{C}$. Finally, $1 \mu \mathrm{l}(200 \mathrm{U} / \mu \mathrm{L}) \mathrm{M}-\mathrm{MLV}$ RT enzyme was added and samples were incubated for 50 minutes, for cDNA synthesis. The purity of the cDNA sample was confirmed by measuring the absorbance at 260/280 in a NanoDrop ND2000, and sample integrity was verified by agarose gel electrophoresis and PCR. Samples were frozen at $-20^{\circ} \mathrm{C}$ for subsequent use in qPCR.

\section{Parasite load estimation by LINJ31 quantitative PCR (qPCR)}

Quantitative real time PCR (qPCR) was performed using the TaqMan ${ }^{\circledR}$ probe $5^{\prime}$ CCT CCT TGG ACT TTG C3' (double-labeled with FAM at the 5'-end and a non-fluorescent quencher at the 3 '-end), and the primers LINJ31F (5'CCG CGT GCC TGT CG3') and LINJ31R (5'CCC ACA CAA GGA GCG ACT3'), which amplify L. (L.) infantum hypothetical protein (partial mRNA; GeneBank accession number LinJ31.1310). Reactions were performed in a 7500 Real Time PCR System (Applied Biosystems), and reaction mixtures contained $3 \mu \mathrm{L}$ of DNA or cDNA samples (or control samples, see below), $10 \mu \mathrm{L}$ of 2X TaqMan Universal PCR Master Mix, $1 \mu \mathrm{L}$ of a mixture of forward

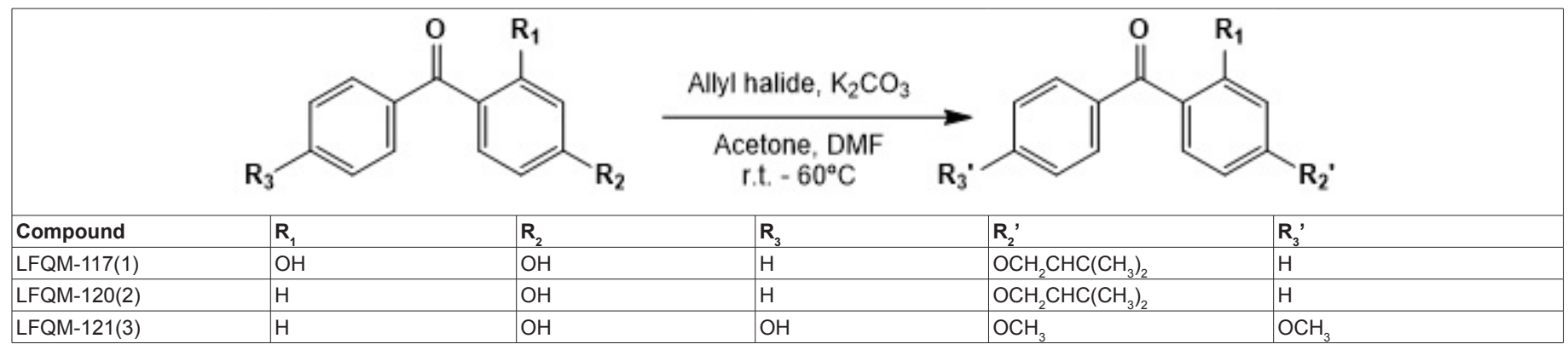

Table 1: Chemical data for benzophenone derivatives 2-Hydroxy-4-O-(3,3-dimethyl)-allylbenzophenone (LFQM-117 (1)), 4-O-(3,3-Dimethyl)-allylbenzophenone (LFQM120 (2)) and 4,4'-Di-methoxybenzophenone (LFQM-121 (3)). 
(LINJ31F) and reverse (LINJ31R) primers (at a concentration of 18 $\mu \mathrm{M})$, and $5 \mu \mathrm{M}$ of the labeled TaqMan probe, in a final volume of $20 \mu \mathrm{L}$. For negative and positive controls, water or DNA extract from $L$. (L.) infantum chagasi (MHOM/BR/1972/BH46 were added, respectively. The following PCR conditions were used: one step of $50^{\circ} \mathrm{C}$ for $2 \mathrm{~min}$, followed by one step of $95^{\circ} \mathrm{C}$ for 10 minutes, and 40 cycles of $95^{\circ} \mathrm{C}$ for $15 \mathrm{~s}$ and $60^{\circ} \mathrm{C}$ for 1 minute. The number of parasites per gram of spleen or liver tissue was calculated based on the linear regression data from the standard curve performed with promastigote DNA. Statistical analysis was performed by Student's t-test with Mann-Whitney (unpaired, twotailed) for the significance test $(\mathrm{p}<0.05)$.

\section{Results and Discussion}

Current therapy against visceral leishmaniasis, based on the use of pentavalent antimonials, is highly toxic and requires a long treatment regimen. Thus, testing of new compounds with leishmanicidal activity is of paramount importance to improve disease treatment and control. Recently, we described the synthesis of benzophenones derivatives with potent activity against Leishmania (L.) amazonensis in vitro $[5,6]$. In this work, we evaluated the anti-parasitic effect of the benzophenone derivatives 1-3 in vivo in hamsters with established infection (50 days post-inoculation) with $L$. (L.) infantum chagasi. For sensitive and accurate quantification of parasites in infected tissues, we used a quantitative reverse transcription PCR (qRT-PCR) assay based on the detection of the LINJ31 marker (Linj31-qPCR), which was used successfully for the quantification of live amastigotes of Leishmania (L.) infantum chagasi $[4,9,15,16]$. The use of RNA as a sample, rather than DNA, minimizes the detection of residual DNA from dead parasites $[8,16]$. Similar qPCR-based tests allow effective quantification of parasite genetic material from the spleen and liver of infected hamsters $[4,9]$ and yield reliable estimates of the parasite burden per gram of tissue $[9,17]$.

To confirm that the system allows linear detection of parasites in infected tissue samples, we produced a standard curve using serial dilutions of DNA from cultured promastigotes of the standard strain of $L$. (L.) infantum chagasi (MHOM/BR/1972/BH46) (Figure 1). The standard curve showed robust linearity and reproducibility $\left(R^{2}=0.9925\right.$, slope $=-3.139$, and $\mathrm{P}$ value $<0.0001)$, with a strong correlation between $\mathrm{Ct}$ values and the number of parasites used to prepare DNA samples. We also determined the detection limit of the system (data not shown), which corresponded to a Ct value of 41.52 . To evaluate the anti-parasitic effect of compounds 1-3, hamsters with established leishmanial infection (50 days after parasite inoculation) were treated for ten days with $50 \mathrm{mg} / \mathrm{kg} / \mathrm{day}$ (in oral suspension) of each test compound, and the spleen and liver of infected animals were collected for RNA extraction, cDNA generation by reverse transcription and qPCR analysis (qRTPCR). The number of amastigotes per gram of (spleen or liver) tissue (Figure 2) was then calculated based on the linear regression parameters obtained from the standard curve (Figure 1).

In the untreated group (UTG; vehicle-treated), the average number of parasites was 1.16 and $0.176 \times 10^{6}$ per gram of tissue, in the spleen and liver, respectively, confirming that these animals had an established infection (clinical symptoms such as ascites and alopecia) with $L$. (L.) infantum chagasi. Treatment with compound LFQM-117 (1) reduced significantly the number of amastigotes in the spleen (to $1.64 \times 10^{2} / \mathrm{g}$ ) when compared with the UTG $(\mathrm{p}<0.05)$ (Figure 2). In contrast, treatment with compound LFQM-120 (2) reduced the number of amastigotes in the liver of infected hamsters (to $1.28 \times 10^{4} / \mathrm{g} ; \mathrm{p}<0.05$ vs. UTG), with only a moderate (albeit statistically significant; $\mathrm{p}<0.05$ ) decrease in parasite numbers in the spleen (to $5.38 \times 10^{4} / \mathrm{g}$ ), when compared with the UTG group (Figure 2) and compound LFQM-121 (3) did not show statistically significant differences in parasite numbers in the spleen or liver of infected animals (Figure 2).

In addition, we observed a potent leishmanicidal activity towards promastigotes in vitro, with $\mathrm{IC}_{50}$ values of $23.66 \mu \mathrm{M}(\mathbf{1}) 27.73 \mu \mathrm{M}$ (2) and $88.02 \mu \mathrm{M}(3)$ [5] and the best selectivity index in relation to amastigote form, which the values were 6.7 (1); 11.9 (2) and 7.3 (3) [6]. It seems that there is a close relationship between the increase of lipophilicity of the compounds with the absorption rates and action in

\section{L. (L.) infantum chagasi (MHOM/BR/1972/BH46)}

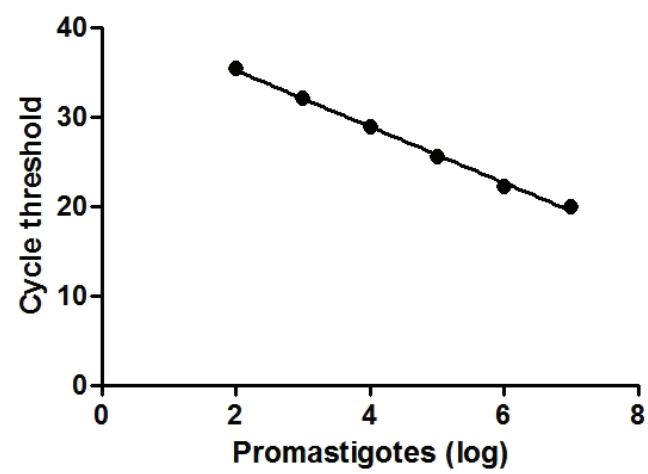

Figure 1: Linj31 quantitative PCR (Linj31-qPCR) standard curve showing cycle threshold $(\mathrm{Ct})$ values versus the number of Leishmania (L.) infantum chagasi promastigotes in samples, as estimated from the original number of parasites used for template DNA extraction. Samples represent serial dilutions (3 $\mu \mathrm{L}$ DNA/reaction)

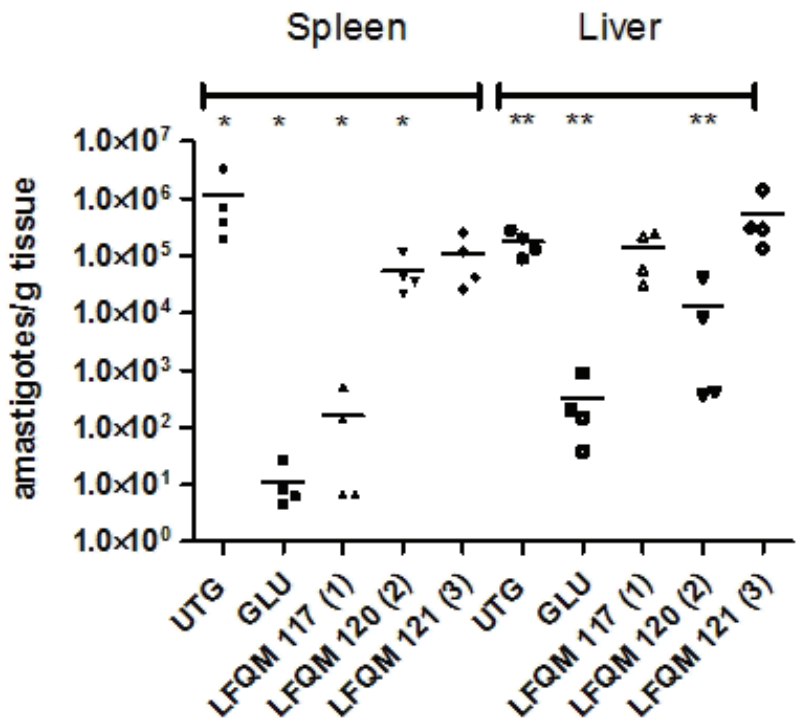

Figure 2: Quantification of parasite burden in the spleen and liver from hamsters infected with Leishmania (L.) infantum chagasi, as quantified by quantitative reverse transcription PCR (qRT-PCR) for the detection of the Linj31marker. The numbers of parasites (amastigotes) per gram of tissue were estimated based on a qPCR standard curve using promastigotes. UTG untreated group (vehicle-treated), GLU, animals treated with Glucantime (50 $\mathrm{mg} / \mathrm{kg} /$ day); compounds LFQM-117 (1); LFQM-120 (2) and LFQM 121 (3), groups treated with the compounds of the same name $(50 \mathrm{mg} / \mathrm{kg} /$ day $) . n=4$ animals/group. Data are represented group mean and individual values for each animal. 
different routes [18,19]. The higher lipophilicity of compounds $\mathbf{1}$ and $\mathbf{2}$ in comparison to compound $3(\mathrm{Q} \log \mathrm{P}=5.29,4.73$ and 3.57 , respectively) is expected to facilitate drug penetration into host cells and parasites, and may underlie the increased activity of these molecules both in vivo (in the liver and spleen, for compounds 2 and $\mathbf{1}$, respectively), and in vitro, towards promastigotes. Recently, de Almeida et al. observed that in vitro activities of compounds $\mathbf{1}$ and $\mathbf{2}$ against cysteine proteases from Leishmania were higher than that of compound 3, which may contribute to the higher in vivo activities of $\mathbf{1}$ and $\mathbf{2}$ in vivo.

To check the validity of current anti-leishmanial treatment, we also treated one group of infected animals with Glucantime (meglumine antimoniate) as reference compound, since this pentavalent antimonial represents the current first-line of treatment against VL [18]. In a recent study, Pinto et al. used a similar methodology to evaluate the leishmanicidal activity of $\mathrm{H} 1$ histamine receptor antagonists, drugs belonging to therapeutic classes such as antiallergics and anxiolytics. Unlike the present work, the standard drug used for the experimental treatment was Miltefosine.

Glucantime $(50 \mathrm{mg} / \mathrm{kg} /$ day, intraperitoneal route) was the most effective compound at reducing the parasite burden in infected animals, since treatment with this drug led to a reduction in the number of amastigotes to 1.15 and $0.32 \times 10^{2}$, in the spleen and liver, respectively (Figure 2). Although the same dosage was used for the treatment with all compounds $(50 \mathrm{mg} / \mathrm{kg} /$ day), Glucantime was administered intraperitoneal, while the test compounds 1, 2 and 3 were administered orally. Theoretically, soluble compounds are totally absorbed in intravenous route but this absorption rate does not apply to others routes. Thereby, different exposure routes can show different effects because the actually absorbed dose is very different from the administered dose. The nature of the compounds can lead different absorption rates being faster to compounds in solution than undissolved suspensions. Furthermore, the biotransformation process after oral administration is distinct from the other routes due the firstpass effect in intestine [20]. This difference in administration route may have contributed to the increased efficacy of Glucantime relative to the other compounds, and it remains possible that the anti-parasitic effect of compounds 1, 2 and 3 would be improved by increasing the concentration of compounds or changing the therapeutic route.

\section{Conclusion}

The higher activities of benzophenone derivatives $\mathbf{1}$ and $\mathbf{2}$ against L. (L.) infantum chagasi in vivo (in comparison to compound $\mathbf{3}$ were compatible to the previously observed in vitro leishmanicidal activity and may be improved by increasing the concentration of compounds in each dose, changing the therapeutic regimen by increasing the number of treatment days, treating two or more times a day, or a combination of them. The pentavalent antimonial Glucantime was the most effective in the treatment of hamsters infected with $L$. (L.) infantum chagasi, but this drug is known by its high toxicity, and despite less potent, benzophenone derivatives such as compounds $\mathbf{1}$ and $\mathbf{2}$ could be interesting prototypes for the development of new effective and safe drug candidates. Further studies are now required to explore different therapeutic regimen and toxicity, and additional immunological and pharmacological assays could clarify the mode of action of compounds these benzophenone derivatives against Leishmania.

\section{Acknowledgements}

The authors are grateful to the Brazilian Agencies FAPEMIG (CBBAPQ-02149/12), CNPq, CAPES, FINEP and UNFAL for financial support and fellowships. We are also grateful to Dr. André Gustavo Tempone (Adolfo Lutz Institute) for providing Glucantime and Dr. Juliana Quero Reimão (UNIFESP) for technical support.

\section{References}

1. Donadeu M, Lightowlers MW, Fahrion AS, Kessels J, Abela-Ridder B (2016) Leishmaniasis in high-burden countries: an epidemiological update based on data reported in 2014. Relev Epidemiol Hebd 91: 73-81.

2. Kobets T, Grekov I, Lipoldova M (2012) Leishmaniasis: Prevention, Parasite Detection and Treatment. Curr Med Chem 19: 1443-1474.

3. Ready PD (2014) Epidemiology of visceral leishmaniasis. Clin Epidemiol 6 : 147-154.

4. Reimão JQ, Colombo FA, Pereira-Chioccola VL, Tempone AG (2012) Effectiveness of liposomal buparvaquone in an experimental hamster model of Leishmania (L.) infantum chagasi. Exp Parasitol 130: 195-199.

5. Maciel-Rezende CM, de Almeida L, Costa ÉD, Pires FR, Alves KF, et al. (2013) Santos, Synthesis and biological evaluation against Leishmania amazonensis of a series of alkyl-substituted benzophenones. Bioorganic Med Chem 21 : 3114-3119.

6. de Almeida L, Alves KF, Maciel-Rezende CM, Jesus LD, Pires FR, et al. (2015) Benzophenone derivatives as cysteine protease inhibitors and biological activity against Leishmania (L.) amazonensis amastigotes. Biomed Pharmacother 75 : 93-99.

7. Reis LE, Coura-Vital W, Roatt BM, Bouillet LÉ, Ker HG, et al. (2013) Molecular diagnosis of canine visceral leishmaniasis: A comparative study of three methods using skin and spleen from dogs with natural Leishmania infantum infection. Vet Parasitol 197: 498-503.

8. Colombo FA, Pereira-Chioccola VL, da Silva Meira C, Motoie G, Gava R, et al. (2015) Performance of a real time PCR for leishmaniasis diagnosis using a L. (L.) infantum hypothetical protein as target in canine samples. Exp Parasito 157: 156-162.

9. Reimão JQ, Colombo FA, Pereira-Chioccola VL, Tempone AG (2011) In vitro and experimental therapeutic studies of the calcium channel blocker bepridil detection of viable Leishmania (L.) chagasi by real-time PCR. Exp Parasitol 128: $111-115$.

10. Reimão JQ, Trinconi CT, Yokoyama-Yasunaka JK, Miguel DC, Kalil SP, et al. (2013) Uliana, Parasite burden in Leishmania (Leishmania) amazonensisinfected mice: Validation of luciferase as a quantitative tool. $\mathrm{J}$ Microbiol Methods 93: 95-101

11. Srivastava A, Sweat JM, Azizan A, Vesely B, Kyle DE (2013) Real-time PCR to quantify Leishmania donovani in hamsters. J Parasitol 99: 145-150.

12. Yang SP, Han LJ, Wen AP, Wang DQ (2009) 4-Methyl-1,3-bis-(3,4-methylenedioxy-benz-yl)-2-(3,4-methyl-enedioxy-phen-yl)imidazolidine. Acta Crystallogr Sect E: Struct Rep Online 65: o3049.

13. Brasil. Ministério da Saúde. Secretaria de Vigilância em Saúde. Departamento de Vigilância Epidemiológica., Manual de Vigilância e Controle da Leishmaniose Visceral, 2014.

14. National Research Council Committee (2011) Guide for the Care and Use of Laboratory Animals. National Academies Press, Washington DC, USA.

15. Stauber LA, Franchino EM, Grun J (1958) An Eight-day Method for Screening Compounds against Leishmania donovani in the Golden Hamster. J Eukaryot Microbiol 5: 269-273.

16. Colombo FA, Odorizzi RM, Laurenti MD, Galati EA, Canavez F, et al. (2011) Detection of Leishmania (Leishmania) infantum RNA in fleas and ticks collected from naturally infected dogs. Parasitol Res 109: 267-274

17. Reithinger R, Dujardin JC (2007) Molecular diagnosis of leishmaniasis: current status and future applications. J Clin Microbiol 45: 21-25.

18. Croft SL, Barrett MP, Urbina JA (2005) Chemotherapy of trypanosomiases and leishmaniasis. Trends Parasitol 21: 508-512.

19. Pinto EG, da Costa-Silva TA, Tempone AG (2014) Histamine H1-recepto antagonists against Leishmania (L.) infantum: An in vitro and in vivo evaluation using phosphatidylserine-liposomes. Acta Trop 137: 206-210.

20. Ning ZH, Long S, Zhou YY, Peng ZY, Sun YN, et al. (2015) Effect of exposure routes on the relationships of lethal toxicity to rats from oral, intravenous intraperitoneal and intramuscular routes. Regul Toxicol Pharmacol 73: 613-619. 